\title{
Microbiological and Clinical Significance of a New Property of Defective Lysis in Clinical Strains of Pneumococci
}

\author{
E. Tuomanen, H. Pollack*, A. Parkinson, \\ M. Davidson, R. Facklam, R. Rich, and O. Zak
}

\author{
From the Rockefeller University, New York, New York; the \\ Arctic Investigations Laboratory, Centers for Disease \\ Control, Anchorage, Alaska; the Division of Bacterial \\ Diseases, Centers for Disease Control, Atlanta, Georgia; \\ and CIBA-GEIGY Limited, Basel, Switzerland
}

\begin{abstract}
A pneumococcal isolate that caused relapsing meningitis in a patient infected with human immunodeficiency virus (HIV) was found to display an unusual response to penicillin - rapid death but a striking lack of cellular lysis. This lytic defect was also detected in all four pneumococcal isolates from three additional HIV-infected patients and in more than half of the clinical isolates from patients with bacteremia. In a rabbit model of meningitis, the lysis-defective strain remained cryptic, with a delay of $5 \mathrm{~h}$ in the onset of leukocytosis in cerebrospinal fluid. A marked burst of leukocytosis was associated with ampicillin-induced lysis of a lysis-sensitive strain but not of a lysis-defective strain. Pneumococcal clinical isolates have different lytic responses to penicillin; defective lysis may adversely affect the course of meningitis, an observation suggesting that autolysins play a role in modulating infectious diseases.
\end{abstract}

A nine-month-old child infected with human immunodeficiency virus (HIV) developed relapsing pneumococcal meningitis. After characterizing the strain, we found an unusual response to penicillin - the bacterium died rapidly but did not undergo cellular lysis. Classically, two responses to penicillin have been described [1,2] for clinical isolates of pneumococci. Typically, the isolates lyse and die rapidly; rarely, lysis is nearly absent, and killing is substantially slower. We characterized in detail the dissociation of killing and lysis in the strain from this patient. This report also describes the discovery of clinical isolates of pneumococci that do not lyse but that undergo rapid loss of viability during penicillin treatment. Addi-

Received for publication 5 October 1987 and in revised form 12 January 1988.

This work was presented in part at the 27th Interscience Conference on Antimicrobial Agents and Chemotherapy, held in New York, New York, on 4-7 October 1987.

This work was supported in part by a Career Investigator Award to Dr. Tuomanen from the Irma T. Hirschl Trust and by grant AI-16794 from the National Institute of Allergy and Infectious Diseases.

We thank J. Kadurugamuwa and B. Hengstler for determining the growth rate of strain $\mathrm{Br}$ in vivo; $\mathrm{E}$. Kovacs for technical assistance; and $\mathbf{M}$. Geller for secretarial assistance.

Please address requests for reprints to Dr. Elaine Tuomanen, The Rockefeller University, 1230 York Avenue, New York, New York 10021.

*Present address: Department of Pediatric Infectious Diseases, New York University, New York, New York. tionally, our studies analyze the impact of the lack of drug-induced lysis (independent of bacterial killing) on the clinical course of pneumococcal infections. We present evidence that autolysins may play a role in modulating the course of some infectious diseases.

\section{Materials and Methods}

Bacterial strains and growth conditions. We used the following strains: encapsulated clinical strains 8249, S SII, Va2, Va3, and Va7 and unencapsulated laboratory strain R6 and its tolerant transformant lyt 4-4; the strains have previously been described [1-3]. Six clinical isolates from the pre-penicillin era $[4,5]$ were obtained from the Centers for Disease Control (Atlanta): SP-88, 96, 99, 108, 142, and 144 (MICs of penicillin, $0.01 \mu \mathrm{g} / \mathrm{mL}$ ). Thirty-three isolates from blood or CSF were provided by the Arctic Investigations Laboratory (Anchorage, Alaska) as part of a retrospective study of invasive pneumococcal disease in Southwest Alaska from 1980 to 1986. Serotypes included types 14 (13 strains), 19A ( 9 strains), 04 (4 strains), 23F ( 2 strains), and 1 strain each of types 10A, 6B, 07F, 33F, and 18C; MICs of penicillin ranged from 0.01 to $0.25 \mu \mathrm{g} / \mathrm{mL}$. One isolate was from an Alaskan infant with an immunoglobulin deficiency and persistent meningitis. Five isolates, including the index strain $\mathrm{Br}$, were obtained from four HIV-infected patients with pneumococ- 
cal bacteremia or meningitis at New York Hospital (New York; serotypes 19, 6, and 9; MICs of penicillin, $0.02 \mu \mathrm{g} / \mathrm{mL}$, including strain $\mathrm{Br}$ ). Three of these isolates caused relapsing disease in two patients: strain $\mathrm{Br}$ caused meningitis that was controlled after two 14-d courses of penicillin, and strains GI and GII caused recurrent bacteremia. Strain $\mathrm{Br}$ was tested in the first passage and in $\leqslant 10$ passages from frozen stock. MICs were determined by using the macrotube dilution method. Identification was made on the basis of growth and morphology on blood-agar plates and of sensitivity to optochin. All strains except the autolysin-deficient strain lyt 4-4 were lysed by deoxycholate [2].

Strains were grown in semisynthetic medium supplemented with $0.1 \%$ yeast (Difco, Detroit) extract $(\mathrm{C}+\mathrm{y} ; \mathrm{pH} 8.0[6])$ in a stationary water bath at $37 \mathrm{C}$. All experiments were carried out during exponential-growth phase from back-diluted overnight cultures. Turbidity was monitored by using a SequoiaTurner spectrophotometer (absorbance at $620 \mathrm{~nm}$; Mountainville, Calif). Stocks of cultures were stored in $\mathrm{C}+\mathrm{y}$ medium with $10 \%$ glycerol at $-70 \mathrm{C}$, by using the first or second passage from the original clinical isolate.

Lysis and killing rates. Cultures of all strains were divided in half when the turbidity reached an absorbance of 0.3-0.4; half of the cultures received 10 $\times$ MIC of penicillin. Strains $\mathbf{R 6}$ and $\mathrm{Br}$ were also tested for lysis in the presence of $\leqslant 100 \times$ MIC of penicillin and of $10 \times$ MIC of imipenem $(0.05$ $\mu \mathrm{g} / \mathrm{mL})$, vancomycin $(5 \mu \mathrm{g} / \mathrm{mL})$, or ampicillin $(0.4$ $\mu \mathrm{g} / \mathrm{mL}$ ). Turbidity was monitored for $6 \mathrm{~h}$ and overnight. Viability was determined at 0,2 , and $4 \mathrm{~h}$ after adding penicillin, by diluting samples from each culture in incomplete semisynthetic medium containing penicillinase $(100 \mathrm{U} / \mathrm{mL}$; Becton-Dickinson, Cockeysville, Md) and by pour-plating in tryptic-soy agar (Difco) supplemented with 3\% sterile, defibrinated sheep blood. Colony counts were made after $24 \mathrm{~h}$ of incubation at $37 \mathrm{C}$.

To compare rates of cell wall degradation, we grew strains $\mathrm{R} 6$, lyt 4-4, and $\mathrm{Br}$ overnight in $\mathrm{C}+\mathrm{y}$ medium containing $1 \mu \mathrm{Ci}$ and $5 \mu \mathrm{g}$ of $\left[{ }^{3} \mathrm{H}\right]$ choline $/ \mathrm{mL}(1$ $\mu \mathrm{Ci} / \mathrm{m} M$; Amersham, Boston). Cells were washed by filtration and were grown for an additional $2 \mathrm{~h}$ in nonradiolabeled medium. Half of each culture received $10 \times$ MIC of penicillin, and all cultures were sampled at hourly intervals for $6 \mathrm{~h}$ to determine $\left[{ }^{3} \mathrm{H}\right]$ choline counts remaining in the cell wall (the material precipitated in boiling SDS [7]). In some ex- periments, $1 \mu \mathrm{g}$ of purified pneumococcal autoly$\sin / \mathrm{mL}$ (amidase [8]) was added $30 \mathrm{~min}$ before penicillin. Crude cell sonicates of strain $\mathrm{Br}$ were prepared and assessed for the presence of endogenous autolytic activity in vitro, as previously described [1, 2]. The profile of the penicillin-binding proteins of strain $\mathrm{Br}$ was compared with that of strain $\mathrm{R} 6$ by using published procedures [3].

Meningitis model. Groups of four rabbits were anaesthetized and placed in stereotaxic frames as previously described $[9,10]$. Pneumococcal strains $S_{\text {III }}$ and $\mathrm{Br}$ were grown to logarithmic phase in $\mathrm{C}+\mathrm{y}$ medium, washed, and resuspended in pyrogen-free saline at a concentration of $10^{3} \mathrm{cfu}$ in $0.2 \mathrm{~mL}$. After removal of $0.3 \mathrm{~mL}$ of CSF, the preparations were instilled into the cisterna magna. Cytochemical determinations of CSF were repeated in each animal over a 24-h period to document changes in the density of leukocytes and the concentrations of glucose, protein, and lactic acid [10]. Bacterial titers in CSF were determined on tryptic-soy agar containing 3\% sheep blood. To determine the effect of ampicillin treatment on the course of the disease, we infected groups of four rabbits with each strain but did not sample these groups until $18 \mathrm{~h}$ later, when infection was established. After CSF sampling, half of the animals in each group received $30 \mathrm{mg}$ of ampicillin $/ \mathrm{kg}$ iv as a single bolus dose. Ampicillin levels in CSF reached a maximum $(\sim 0.5 \mu \mathrm{g} / \mathrm{mL}) 30 \mathrm{~min}$ after infusion $\left(>10 \times\right.$ MIC of both $\mathrm{S}_{\mathrm{III}}$ and $\left.\mathrm{Br}\right)$, a result that agreed with previous results [9]. Cytochemical parameters and bacterial titers in CSF were determined 4 and $24 \mathrm{~h}$ after administering ampicillin.

Summaries of clinical data. The medical records for the 30 Alaskan patients and their 156 household members were obtained and reviewed for antibiotic usage for the six months preceding the index pneumococcal infection. The duration of antibiotic usage (median number of days) was recorded and compared by using a nonparametric statistical test. The duration of fever and hospitalization (median number of days) for the 30 invasive pneumococcal illnesses was similarly recorded and analyzed.

\section{Results}

Characteristics of lysis-defective pneumococci. Unlike the standard lysis-sensitive/ fast-kill (R6) and lysis-defective/slow-kill (lyt 4-4) laboratory strains, strain $\mathrm{Br}$ demonstrated an apparent dissociation of lysis and killing (lysis sensitive/fast kill) in response 
a

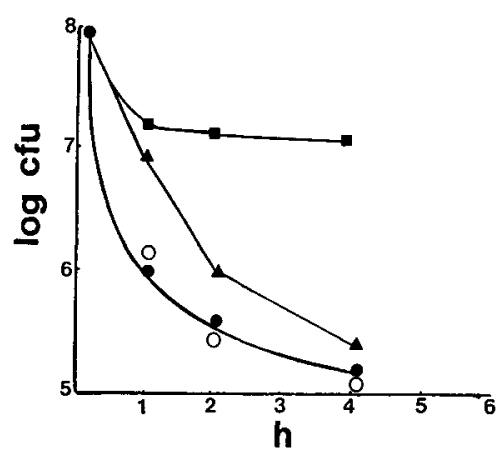

b

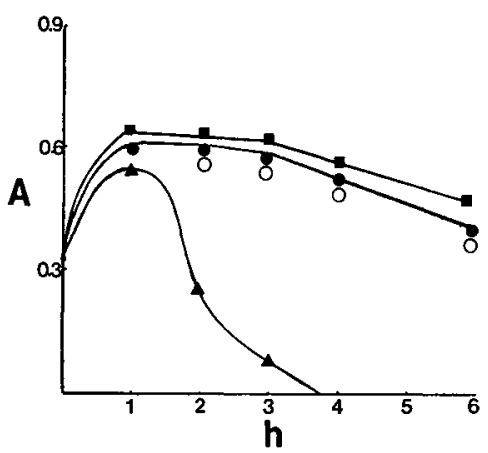

Figure 1. Comparison of the killing $(a)$ and lysis $(b)$ rates of representative strains from each of the three groups of pneumococci. Triangles, lysis-sensitive strain R6; circles, lysis-defective, kill-sensitive strain $\mathrm{Br}$; squares, killresistant (tolerant) strain lyt 4-4; closed symbols, penicillin concentration of 10 $\times$ MIC; and open symbols, penicillin concentration of $100 \times$ MIC. to penicillin (figure 1). Penicillin lysis was minimal, even at $100 \times$ MIC (figure 1, open circles). When rates of cell wall degradation between the penicillinsensitive, lysis-sensitive/fast-kill (R6) strain and strain $\mathrm{Br}$ were compared, cell wall degradation of strain $\mathrm{Br}$ was markedly reduced at both $10 \times$ and $100 \times$ MIC (table 1); this response was also found with ampicillin. Rapid lysis analogous to the R6 standard strain was achieved, however, by two other inhibitors of cell wall synthesis - imipenem and vancomycin. The profile of penicillin-binding proteins for strain $\mathrm{Br}$ (determined at saturating concentrations of the radioactive penicillin) was the same as that for the standard laboratory strain R6 (data not shown).

Crude extracts of strain $\mathrm{Br}$ contained autolytic activity capable of solubilizing $>90 \%$ of $\left[{ }^{3} \mathrm{H}\right]$ lysine label from the cell wall of strain R6 in vitro, a result indicating the presence of active autolysin. This result was consistent with the fact that deoxycholate also caused lysis and cell wall degradation of strain

Table 1. Percent loss of $\left[{ }^{3} \mathrm{H}\right]$ choline label from cell wall $4 \mathrm{~h}$ after treating strains $\mathrm{R} 6$ and $\mathrm{Br}$ with various lytic stimuli.

\begin{tabular}{|c|c|c|c|c|c|}
\hline \multirow[b]{3}{*}{ Strain } & \multicolumn{5}{|c|}{ Lytic stimulus } \\
\hline & \multirow[b]{2}{*}{ None } & \multicolumn{2}{|c|}{$\begin{array}{l}\text { Penicillin } \\
(\times \text { MIC })\end{array}$} & \multirow{2}{*}{$\begin{array}{c}10 \times \mathrm{MIC} \\
\text { of penicillin }+ \\
1 \mu \mathrm{g} \text { of ami- } \\
\text { dase } / \mathrm{mL}^{*}\end{array}$} & \multirow{2}{*}{$\begin{array}{c}0.1 \% \\
\text { deoxycholate }\end{array}$} \\
\hline & & 10 & 100 & & \\
\hline R6 & 9 & 78 & 92 & 92 & 94 \\
\hline $\mathrm{Br}$ & 7 & $17^{\dagger}$ & $34^{\dagger}$ & 87 & 96 \\
\hline
\end{tabular}

* Purified autolysin of strain R6; results with amidase alone were not different from results with no lytic stimulus.

$\dagger P<.01$, as compared with strain R6 by using the Student's $t$ test.
$\mathrm{Br}$ (table 1) and of all the other lysis-defective clinical isolates of pneumococci that we studied (deoxycholate-induced lysis is an autolysin-dependent phenomenon [2]). The presence of deoxycholate-induced lysis and extensive stationary-phase lysis, albeit delayed in onset, also indicated that the cell wall of strain $\mathrm{Br}$ could be hydrolyzed by autologous enzyme. Adding exogenous purified autolysin to cultures of strain $\mathrm{Br}$, then adding $10 \times \mathrm{MIC}$ of penicillin, resulted in lysis and cell wall degradation comparable to that in the lysis-sensitive strain R6 (table 1).

Lysis-defective pneumococci in clinical isolates. Figure 2 compares the lytic and killing responses after exposure to $10 \times \mathrm{MIC}$ of penicillin for the 51 clinical isolates that were studied. The three general types of responses shown in figure 1 were observed: group a, lysis sensitive and fast killed (16 strains, $31 \%$ ); group $b$, lysis defective and fast killed (29 strains, 57\%); and group c, lysis defective and slowly killed ( 6 strains, 12\%). The classic lysis-sensitive response, defined as those isolates showing $>50 \%$ decrease in turbidity after $4 \mathrm{~h}$, was characteristic of the standard laboratory strain R6 and 15 clinical isolates (including strain $\mathrm{S}_{\text {III }}$, two pre-penicillin isolates, and 12 Alaskan strains). The remaining 35 strains were lysis defective $(<50 \%$ decrease in turbidity after $4 \mathrm{~h})$, with a spectrum of slow-lysis rates. This group also demonstrated a wide range of rates and degrees of penicillin-induced killing. For the purposes of comparison, an arbitrary dividing line was established to define fast vs. slow killing, as follows. The wellcharacterized laboratory standard (strain R6) was actually the least kill-sensitive strain in group a (mean, $3.0 \pm 0.6 \log \mathrm{cfu} / \mathrm{mL}$ ). This value minus one standard deviation (i.e., 2.4) was chosen as the cutoff value for "lysis-sensitive and fast-killed" pneu- 


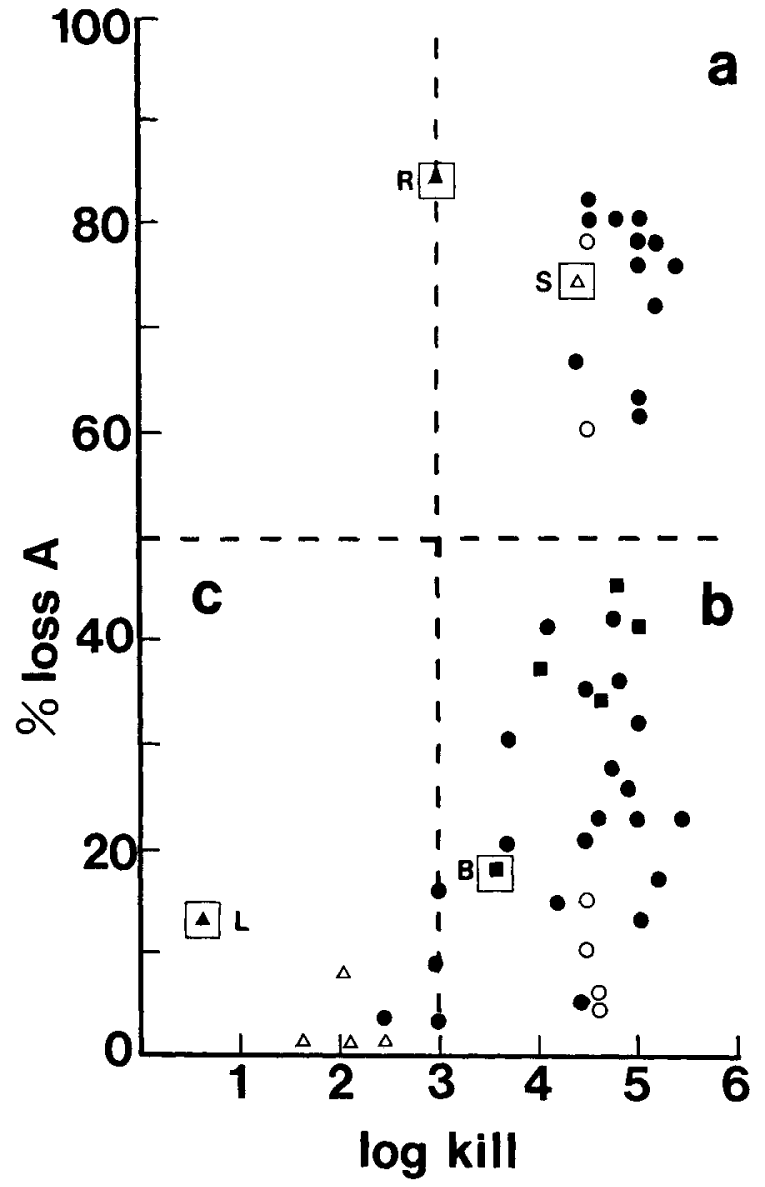

Figure 2. Scattergram comparing percent lysis and log killing of pneumococci $4 \mathrm{~h}$ after administration of $10 \times$ MIC of penicillin. Dashed lines represent borderline values between groups, derived as explained in the text. $A$, lysissensitive, kill-sensitive; $B$, lysis-defective, kill-sensitive; $C$, lysis-defective, kill-resistant (tolerant); $\boldsymbol{\Delta}$, laboratory strains, including R6 $(R)$ and lyt $4-4(L)$; 0 , clinical isolates from Alaska; $\mathbf{D}$, isolates from HIV-infected patients including $\mathrm{Br}(B) ; \Delta$, previously well-characterized clinical isolates, including $S_{I I I}(S)$; and $O$, isolates from the pre-penicillin era.

mococci. On this basis, six strains were classified as "lysis defective" and "slowly killed" (group c): the tolerant laboratory standard strain lyt 4-4; the previously described [1] "tolerant" clinical isolates 8249 ,
$\mathrm{Va} 2, \mathrm{Va} 3$, and Va7; and one Alaskan isolate. Group b, the group with the largest number of strains ( 29 strains, $57 \%$ of the total), was characterized by the novel phenotype "lysis defective" but "fast killed" in response to penicillin. The majority of these strains were as highly kill sensitive ( $>4 \log$ kill after $4 \mathrm{~h}$ ) as were the "normal," lysis-sensitive, fast-kill strains in group a. Four of six isolates from the pre-penicillin era, all five isolates from HIV-infected patients (including strain $\mathrm{Br}$ ), and 20 of the 33 Alaskan isolates were in this lysis-defective group.

Table 2 describes the relation between lytic and killing properties and the MIC and serotype of the 49 clinical isolates. Lysis-sensitive, kill-sensitive strains were always penicillin susceptible (MIC $<0.1$ $\mu \mathrm{g} / \mathrm{mL}$ ), and most strains were type 14 . Tolerant (lysis-defective, kill-resistant) strains were always intermediately or highly resistant to penicillin (MIC $>0.1 \mu \mathrm{g} / \mathrm{mL}$ ), and all but one (6B) were type 19 . The mean MIC of the lysis-defective group was somewhat elevated, but the strains were nearly evenly distributed above and below an MIC of $0.1 \mu \mathrm{g} / \mathrm{mL}$.

The presence of stationary-phase lysis was highly variable in these strains and was often markedly delayed in onset. No relation between the lytic response to penicillin and stationary-phase lysis was found.

Experimental meningitis due to lysis-sensitive and lysis-defective pneumococci. The lysis-sensitive strain $\mathrm{S}_{\mathrm{III}}$ has been studied extensively in the rabbit model of meningitis $[9,10]$. We compared the growth rate, the onset of CSF abnormalities, and the response to ampicillin of strain $S_{I I I}$ with that of the lysis-defective strain $\mathrm{Br}$. Both strains were penicillinsusceptible (MIC, $0.02 \mu \mathrm{g} / \mathrm{mL}$ ) isolates from children with meningitis, and both strains had a doubling time of $\sim 60 \mathrm{~min}$ in vivo in CSF. For strain $\mathrm{S}_{\text {III }}$, the appearance of $>100$ leukocytes $/ \mu \mathrm{L}$ of CSF occurred $9 \mathrm{~h}$ after infection at a density of $7 \times 10^{5}$ $\mathrm{cfu} / \mathrm{mL}$; in contrast, strain $\mathrm{Br}$ induced leukocytosis only at $14 \mathrm{~h}$ after infection at a density of $7 \times 10^{7}$ $\mathrm{cfu} / \mathrm{mL}$. At $24 \mathrm{~h}$ after infection the bacterial den-

Table 2. Relation of lytic and killing properties to MIC and serotype of clinical strains.

\begin{tabular}{lcccccc} 
& \multicolumn{2}{c}{ MIC of penicillin $(\mu \mathrm{g} / \mathrm{mL})$} & & \multicolumn{2}{c}{ No. of strains } \\
\cline { 2 - 3 } Group & Mean $\pm \mathrm{SD}$ & Range & & Total & Serotype 14 & Serotype 19 \\
\hline Lysis sensitive/kill sensitive & $0.026 \pm 0.01$ & $0.015-0.06$ & 15 & 11 & 1 \\
Lysis defective/kill sensitive & $0.095 \pm 0.08$ & $0.02-0.25$ & 29 & 2 & 8 \\
Lysis defective/kill resistant & $2.0 \pm 2.3$ & $0.2-6.0$ & 5 & 0 & 4 \\
\hline
\end{tabular}


Table 3. Comparison of CSF cytochemistry and bacterial titers, $4 \mathrm{~h}$ after ampicillin therapy, of lysis-sensitive $\left(\mathrm{S}_{\mathrm{III}}\right)$ and lysis-defective $(\mathrm{Br})$ pneumococci in a rabbit model of meningitis.

\begin{tabular}{|c|c|c|c|c|c|}
\hline \multirow[b]{3}{*}{ Treatment group } & \multirow{3}{*}{$\begin{array}{l}\text { Strain (no. } \\
\text { of animals) }\end{array}$} & \multicolumn{4}{|c|}{ CSF cytochemistry* } \\
\hline & & \multicolumn{3}{|c|}{ No. (mean \pm SD) of leukocytes at } & \multirow{2}{*}{$\begin{array}{c}\text { Change in } \\
\text { bacterial titer } \\
\text { (mean } \pm \mathrm{SD} \\
\log \mathrm{cfu} / \mathrm{mL})\end{array}$} \\
\hline & & $18 \mathrm{~h}$ & $22 \mathrm{~h}$ & change & \\
\hline \multirow[t]{2}{*}{ Control } & $\mathrm{S}_{\text {III }}(6)$ & $2647 \pm 2606$ & $5860 \pm 4025$ & +221 & $+1.5 \pm 0.8$ \\
\hline & $\mathrm{Br}(8)$ & $2775 \pm 3616$ & $1630 \pm 1527$ & -59 & $+2.5 \pm 1.2$ \\
\hline \multirow[t]{2}{*}{ Ampicillin } & $S_{\text {III }}(7)$ & $2651 \pm 1678$ & $19202 \pm 8511$ & $+724^{\dagger}$ & $-3.1 \pm 0.9$ \\
\hline & $\mathrm{Br}(8)$ & $3147 \pm 2838$ & $7370 \pm 6929$ & +234 & $-1.9 \pm 1.1$ \\
\hline
\end{tabular}

* Treatment was initiated at $18 \mathrm{~h}$ of infection.

$\dagger P<.01$ when compared with control.

sity of strain $\mathrm{Br}$ exceeded the density of $\mathrm{S}_{\mathrm{III}}$ by 2 logs. At the same time, however, the leukocyte density and protein concentrations for strain $\mathrm{Br}$ remained approximately one-third that of $\mathrm{S}_{\text {III }}$.

Table 3 compares the course of meningitis due to strains $\mathrm{S}_{\mathrm{III}}$ and $\mathrm{Br}$ after treatment with ampicillin. Without treatment, bacterial counts increased 2 logs between 18 and $22 \mathrm{~h}$ for both strains, yet the leukocyte density for strain $S_{\text {III }}$ doubled and that for strain $\mathrm{Br}$ decreased to approximately one-half. In ampicillin-treated animals, both strains were killed, and the change in bacterial titer was 2-3 logs $(P$ $>0.1$ ). The number of leukocytes appearing in the CSF $4 \mathrm{~h}$ after the onset of lysis of strain $\mathrm{S}_{\text {III }}$ was, however, a mean of six times greater than the values before ampicillin, whereas the corresponding value for the lysis-defective strain $\mathrm{Br}$ was only doubled ( $P$ $<.001$ ). Twenty-four hours after ampicillin, bacterial titers in animals infected with strain $\mathrm{Br}$ all rebounded to $>3$ logs, and all the animals died within $72 \mathrm{~h}$. Three-fourths of the animals infected with strain $\mathrm{S}_{\mathrm{III}}$ showed an increase in bacterial titers, but the maximum value was only $\sim 1 \log$, and $50 \%$ of these animals survived (i.e., alive at seven days).

Previous antibiotic exposure and clinical course of patients with pneumococcal bacteremia. Table 4 compares the antibiotic exposure, before the index infection, of 30 Alaskan patients who had pneumococcal bacteremia; 12 patients had lysis-sensitive strains, and 18 had lysis-defective strains. No significant difference could be detected between the median number of days of antibiotic usage by the two groups for three to six months before hospitalization, a result that eliminated any possible antibioticrelated selection for the lysis defect. In fact, more patients with lysis-defective than lysis-sensitive infections did not receive any penicillin (8 of 18 vs. 1 of $12 ; P<.05)$. A review of the medical records for

Table 4. Previous antibiotic exposure and clinical course of 30 patients with pneumococcal bacteremia.

\begin{tabular}{|c|c|c|c|}
\hline \multirow[b]{2}{*}{ Therapy } & \multicolumn{3}{|c|}{ Pneumococcal response to penicillin } \\
\hline & $\begin{array}{l}\text { Lysis sensitive } \\
\quad(n=12)\end{array}$ & $\begin{array}{l}\text { Lysis defective } \\
\qquad(n=18)\end{array}$ & $P$ \\
\hline \multicolumn{4}{|l|}{ Antibiotic exposure* } \\
\hline Days of penicillin therapy & $20.5(0-70)$ & $6.0(0-42)$ & .06 \\
\hline Courses of penicillin therapy & $2.5(0-7)$ & $1.5(0-5)$ & .09 \\
\hline Days of all $\beta$-lactam therapy & $20.5(0-70)$ & $15.5(0-22)$ & .19 \\
\hline Courses of all $\beta$-lactam therapy & $2.5(0-7)$ & $2.0(0-8)$ & .20 \\
\hline Days of all other antibiotic therapy & $25.0(0-133)$ & $10.0(0-160)$ & .78 \\
\hline \multicolumn{4}{|l|}{ Clinical course of index infection } \\
\hline Days in hospital & $3.5(0-6)$ & $3.5(0-14)$ & NS \\
\hline Days of temperature $>37.2 \mathrm{C}$ & $2.0(0-4)$ & $3.5(0-7)$ & NS \\
\hline
\end{tabular}

NOTE. Data are median no. (range); NS $=$ not significant, $n=$ no. of patients.

* Antibiotic exposure during the six months before the index episode of pneumococcal bacteremia. 
all other members of households in which there was a case of invasive pneumococcal infection showed no difference in previous antibiotic usage.

The data on the clinical courses of the HIVinfected patients and the Alaskan patients with bacteremia were available for retrospective study. Two of the four HIV-infected patients and one immunoglobulin-deficient child experienced relapsing or persistent disease; all of the causative pneumococcal strains were lysis defective. In particular, strain $\mathrm{Br}$, which caused relapsing meningitis in one of the HIVinfected patients, was eradicated only after tandem, 14-day courses of penicillin and ampicillin. Meningitis persisted in the immunoglobulin-deficient child after $18 \mathrm{~d}$ of penicillin. In contrast, in normal, immunocompetent hosts, there were no differences detected between the patients with lysis-sensitive and lysis-defective strains after comparing days of hospitalization or days with fever $(>37.2 \mathrm{C})$, an observation suggesting that the lytic phenotype did not influence the course of bacteremia (table 4).

\section{Discussion}

Our study shows that the response to penicillin in clinical isolates of pneumococci varies considerably with respect to bacterial lysis. Recently $[1,11]$, it has become evident that decreased susceptibility to killing, i.e., tolerance, is increasing in frequency among clinical isolates of pneumococci. We now report that susceptibility to lysis is also changing in pneumococci, and these properties may be independent variables. Thus, it is possible to divide pneumococci into three general response patterns to penicillin: group a, both lysis and kill sensitive; group b, lysis defective but kill sensitive; and group c, both relatively lysis and kill resistant. The response to penicillin of pneumococci in group a typifies the normal response that has been observed in the laboratory strains extensively used over the past decades, and the response of pneumococci in group $c$ characterizes a tolerant response. The existence of group b pneumococci, in which lysis and killing are dissociated, suggests that bacterial death and lysis occur by different mechanisms in clinical isolates of pneumococci. Killing without lysis and cell wall degradation are properties that characterize most strains of Lancefield group A streptococci $[12,13]$. Penicillin-resistant pneumococci have previously been shown [1] to be lysis defective, but they also show a defect in killing, a result that is much more extensive than that described in figure 2 (compare open triangles in group $c$ and closed circles in group b). Our data indicate that the combination of defective lysis and kill sensitivity is not rare among clinical strains, because more than one-half of the strains we tested demonstrated this trait. The actual prevalence of the lysis defect, with or without alteration of sensitivity to killing, cannot be determined from our data.

The lysis-defective pneumococci cannot be easily recognized in the microbiology laboratory unless they are characterized by using lysis-and-kill curves. It is important to understand that a lytic defect cannot be used as evidence for a kill defect in the clinical microbiology laboratory. The lysis-defective trait is clearly compatible with MICs characteristic of highly sensitive ( $\leqslant 0.02 \mu \mathrm{g} / \mathrm{mL}$ ) bacteria. The selection pressure that is operative in generating lytic defects in clinical isolates is not known. Recent laboratory experiments [14] have demonstrated that when penicillin is used in a cyclic (greater than the MIC to less than the MIC) manner it selects for lysisdefective pneumococci. This type of antibiotic pressure characterizes antibiotic usage in the clinical setting. Among the isolates collected from bacteremic patients, however, the occurrence of antibiotic therapy in both individuals and households before infection was not more common in the patients with lysis-defective than with lysis-sensitive strains. The fact that isolates from the pre-penicillin era also demonstrate this property suggests that other nonantibiotic selective pressures, e.g., survival in storage, also exist. It should be noted, however, that a storage artifact does not adequately explain the lysisdefective phenotype, because strain $\mathrm{Br}$ and six other lysis-defective strains demonstrated the lysisdefective phenotype after only one passage from the clinical specimen. Additional studies [15] have shown the trait to be genetically transformable.

The mechanism of the lytic defect in strain $\mathrm{Br}$ is similar to that described for the tolerant South African pneumococci [1] and in laboratory constructs of lysis-defective strains [14]. The lytic defect mechanism may involve some aspect of autolysin control, because strain $\mathrm{Br}$ lysed and degraded cell wall in response to deoxycholate, imipenem, and vancomycin, a result indicating that active autolysin and hydrolysis-sensitive cell wall substrate were present. Exogenous wild-type autolysin could depolymerize the cell wall of penicillin-treated, lysis-defective cells; conversely, autolysin extracts of strain $\mathrm{Br}$ could de- 
grade wild-type cell wall in vitro. Thus, qualitatively, the autolytic capacity of the lysis-defective strain $\mathrm{Br}$ appeared to be intact but not triggered by penicillin.

Using a rabbit model of meningitis, we investigated the autolytic defect to determine the clinical context in which tests to detect the phenotype should be done. A rigorous comparison of the course of infection in lysis-defective vs. -sensitive strains would require using isogenic strains of pneumococci, but constructing such strains is difficult at the present time, because no selection technique exists for combining defective lysis with kill sensitivity. Despite this limitation, however, several obvious differences in the course of infection were found. Clearly, infection progressed much further before leukocytes were recruited into the CSF when rabbits were challenged with the lysis-defective strain as opposed to the highly lytic strain. Continued growth of the lysis-defective strain did not result in increasing CSF leukocytosis. The onset of leukocytosis in CSF has occurred between 0.7 and $1 \times 10^{6} \mathrm{cfu} / \mathrm{mL}$ for all pneumococci tested thus far, regardless of capsular type [10]. Thus, a delay of $5 \mathrm{~h}$ and an increase in the bacterial density to $>10^{7} \mathrm{cfu} / \mathrm{mL}$ before the onset of leukocytosis for the lysis-defective strain is an important result. The cryptic nature of infection with the lysisdefective strain would contribute to higher morbidity and mortality from this type of infection. It is known that antibiotic-induced lysis and subsequent release of cell wall-degradation products contribute to generating inflammation in the CNS and the lung $[10,16,17]$. By this criterion, the lysis-defective strains would be expected to generate less inflammation, particularly during antibiotic-induced cell death in vivo. This result was indeed what was found; and this result would be expected to improve the outcome of disease once the cryptic infection was discovered [16]. The rebound in bacterial titers $24 \mathrm{~h}$ after ampicillin was greater in the lysis-defective strains than in the lysis-sensitive strains. Because both lysisdefective and -sensitive strains were killed equally well, this increase may be due to an insufficient number of leukocytes to control a cryptic infection of high bacterial density.

Our results suggest that autolysis plays a role in shaping the course of pneumococcal meningitis in vivo, independent of the sensitivity of the bacterium to antibiotic-induced death. Autolysins can be considered dual-edged swords because when they remain cryptic (lysis defective), recruitment of host defenses to sites of infection outside the blood stream is slower and the rebound in bacterial growth after antibiotics is greater. On the other hand, without lysis, inflammation associated with antibiotic-induced bacterial death is less prominent. There appears to be a negative balance between these effects in experimental meningitis, a system that is highly sensitive to the complications of lysis. Defective lysis, however, may not require longer therapy when bacteremia occurs in normal children. A retrospective comparison of the clinical course of $29 \mathrm{im}$ munocompetent patients with invasive disease caused by lysis-defective strains showed that this property did not promote a relapsing or complicated course for bacteremia. In four HIV-infected patients and one immunoglobulin-deficient patient, however, all six invasive infections were caused by lysisdefective strains; three of the five patients experienced relapsing or persistent disease. This result suggests that a prospective analysis of the prevalence and the impact on the clinical course of lysisdefective pneumococci is warranted in immunocompromised patients and in severe invasive disease such as meningitis in the normal host.

\section{References}

1. Liu HH, Tomasz A. Penicillin tolerance in multiply drugresistant natural isolates of Streptococcus pneumoniae. J Infect Dis 1985;152:365-72

2. Tomasz A, Albino A, Zanati E. Multiple antibiotic resistance in a bacterium with suppressed autolytic system. Nature 1970;227:138-40

3. Handwerger S, Tomasz A. Alterations in penicillin-binding proteins of clinical and laboratory isolates of pathogenic Streptococcus pneumoniae with low levels of penicillin resistance. J Infect Dis 1986;153:83-9

4. Kauffmann F, Morch E, Schmith K. On the serology of the pneumococcus-group. J Immunol 1940;39:397-426

5. Morch E. Further studies on the serology of the pneumococcus group. J Immunol 1942;43:177-202

6. Tomasz A. Cellular metabolism in genetic transformation of pneumococci: requirement for protein synthesis during induction of competence. J Bacteriol 1970;101:860-71

7. Park JT, Hancock R. A fractionation procedure for studies of the synthesis of cell-wall muropeptide and of other polymers in cells of Staphylococcus aureus. J Gen Microbiol 1960;22:249-58

8. Ronda C, Garcia JL, Garcia E, Sanchez-Puelles JM, Lopez $\mathrm{R}$. Biological role of the pneumococcal amidase. Cloning of the lyt $A$ gene in Streptococcus pneumoniae. Eur J Biochem 1987;164:621-4

9. Sande MA, Korzeniowski OM, Allegro GM, Brennan RO, Zak $O$, Scheld WM. Intermittent or continuous therapy of experimental meningitis due to Streptococcus pneumoniae in rabbits: preliminary observations on the postantibiotic effect in vivo. Rev Infect Dis 1981;3:98-109 
10. Tuomanen E, Tomasz A, Hengstler B, Zak O. The relative role of bacterial cell wall and capsule in the induction of inflammation in pneumococcal meningitis. $J$ Infect Dis 1985;151:535-40

11. Handwerger S, Tomasz A. Antibiotic tolerance among clinical isolates of bacteria. Rev Infect Dis 1985;7:368-86

12. Gutmann L, Tomasz A. Pennicillin-resistant and penicillintolerant mutants of group A streptococci. Antimicrob Agents Chemother 1982;22:128-36

13. Horne D, Tomasz A. Tolerant responses of Streptococcus sanguis to beta-lactams and other cell wall inhibitors. Antimicrob Agents Chemother 1977;11:888-96

14. Moreillon P, Tomasz A. Penicillin resistance and defective lysis in clinical isolates of pneumococci: evidence for two kinds of antibiotic pressures operating in the clinical environment. J Infect Dis 1988;157:1150-7
15. Moreillon $\mathrm{P}$, Tomasz A. Tolerance to beta lactam antibiotics (AB) induced by cyclic treatments (ttt) of pneumococci with high concentrations of penicillin (Pen) [abstract no. 1316]. In: Program and abstracts of the 27th Interscience Conference on Antimicrobial Agents and Chemotherapy. Washington, DC: American Society for Microbiology, 1987

16. Tuomanen E, Hengstler B, Rich R, Bray MA, Zak O, Tomasz A. Nonsteroidal anti-inflammatory agents in the therapy for experimental pneumococcal meningitis. J Infect Dis 1987;155:985-90

17. Tuomanen $E$, Rich $R$, Zak $O$. Induction of pulmonary inflammation by components of the pneumococcal cell surface. Am Rev Respir Dis 1987;135:869-74 